\title{
Study of Pleural Effusion in Chronic Kidney Disease
}

\author{
Authors \\ Dr Gadkari Nitin ${ }^{1}$, Dr Rishi Patel ${ }^{2}$, Dr Tushar Shet ${ }^{3}$, Dr Ameya Patil, \\ Dr Sumiran Mahajan \\ ${ }^{1}$ Associate Professor, Bharati Hospital and Research Centre, Pune \\ ${ }^{2}$ Professor, Bharati Hospital and Research Centre, Pune \\ ${ }^{3}$ Post Graduate student, Bharati Hospital and Research Centre, Pune
}

\begin{abstract}
Introduction: Chronic kidney disease is a spectrum of pathophysiological processes and condition which usually presents with fluid overload leading to accumulation of fluid in pleural or peritoneal cavities. Here is an attempt to study the profile of pleural effusion in chronic kidney disease patients.

Methodology: A sample size of 40 patients was taken on OPD and IPD basis and pleural fluid was studied and results were analysed. It was a cross sectional study.

Results: The study showed male predominance. Transudative effusions were noted in $75 \%$ of cases and the most common cause of transudative pleural effusion was cardiac failure and most common presentation was cough. In exudative effusion, tubercular pleural effusions were the most common cause in our series.

Keywords: Chronic kidney disease, pleural effusion, exudative, transudative.
\end{abstract}

\section{Introduction}

Chronic kidney disease (CKD) encompasses a spectrum of different pathophysiologic processes associated with abnormal kidney function and a progressive decline in glomerular filtration rate and is defined as a decrease in the GFR less than $60 \mathrm{ml} / \mathrm{min} / 1.73$ square meters for more than 3 months or presence of 1 or more markers of CKD such as albuminuria, active urinary sediments, fluid and electrolyte imbalance due to tubular cause, abnormal kidneys on radiological examination, abnormal kidneys on renal biopsy finding or post renal transplant status of the patient. $^{(1)}$

Pleural effusion is collection of excess quantity of fluid in the pleural space. With the increase in the number of chronic kidney disease patients', the spectrum of pathologies that follow it are vast and among them, pleural effusion is very important. Pleural effusion in such patients is a common diagnostic dilemma as it may arise from CKD itself (fluid overload, nephritic syndrome, uremic pleurisy), concomitant infections (especially pneumonias and tuberculosis (TB) in our country), pulmonary embolisms or diseases causing pleurarenal syndromes, like systemic lupus erythematosus. $^{(2)}$

In the Indian scenario, in general population, the ratio of occurrence of both exudative and transudative pleural effusion is in the same frequency. ${ }^{(3)}$ However in CKD patients transudative effusion are more than exudative 
effusions. The transudative effusions are majorly secondary to congestive cardiac failure. Among exudative effusion, TB effusion is a major challenge. It was observed that in patients with CKD, uremia was the most common cause of exudative pleural effusion even in a TB-endemic country like India. ${ }^{(4)}$

\section{Methods}

This study was a tertiary hospital based observational study conducted at Department of Medicine, Bharati Hospital, Pune from September 2016 to March 2018. The aim of the study was to evaluate the spectrum of pleural effusion in patients with Chronic Kidney Disease. All 40 consecutive IPD and OPD consenting patients during this study period of 18 months who had CKD with pleural effusion were included in the study. Patients having acute renal failure, concomitant cirrhosis, malignancy and those having very small non tappable effusions were excluded from the study. A detailed history and examination were done in all cases. Pleural fluid was studied for biochemical parameters (protein, LDH), cytology and categorised as transudate or exudates as per Light's criteria. Exudative pleural effusion was studied additionally by Z-N stain, Gram - stain and culture for pyogenic cytology.

\section{Results}

Mean age of the study subjects was 53.67 years with over half of the cases in the age group of 4160 years. A male predominance was seen in the study group with $57.5 \%$ males to $42.5 \%$ females.

Out of total 40 cases, $30(75 \%)$ had transudative effusion while remaining $10(25 \%)$ had exudative effusion.

Out of the 30 cases of transudative effusion, 10 $(33.33 \%)$ were cardiac failure, Hypoalbuminemia - 7(23.33\%), nephrotic-3(10\%), volume overload $2(6.66 \%)$, atelectasis-2(6.66\%), scleroderma $1(3.33 \%)$ and hepatic cirrhosis (3.33\%).

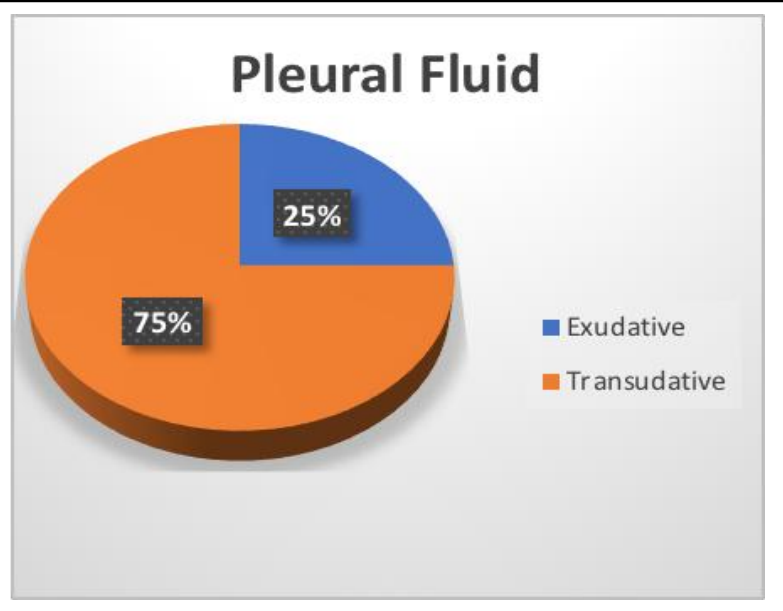

Figure 1. (a) Distribution of cases as per Type of Pleural effusion
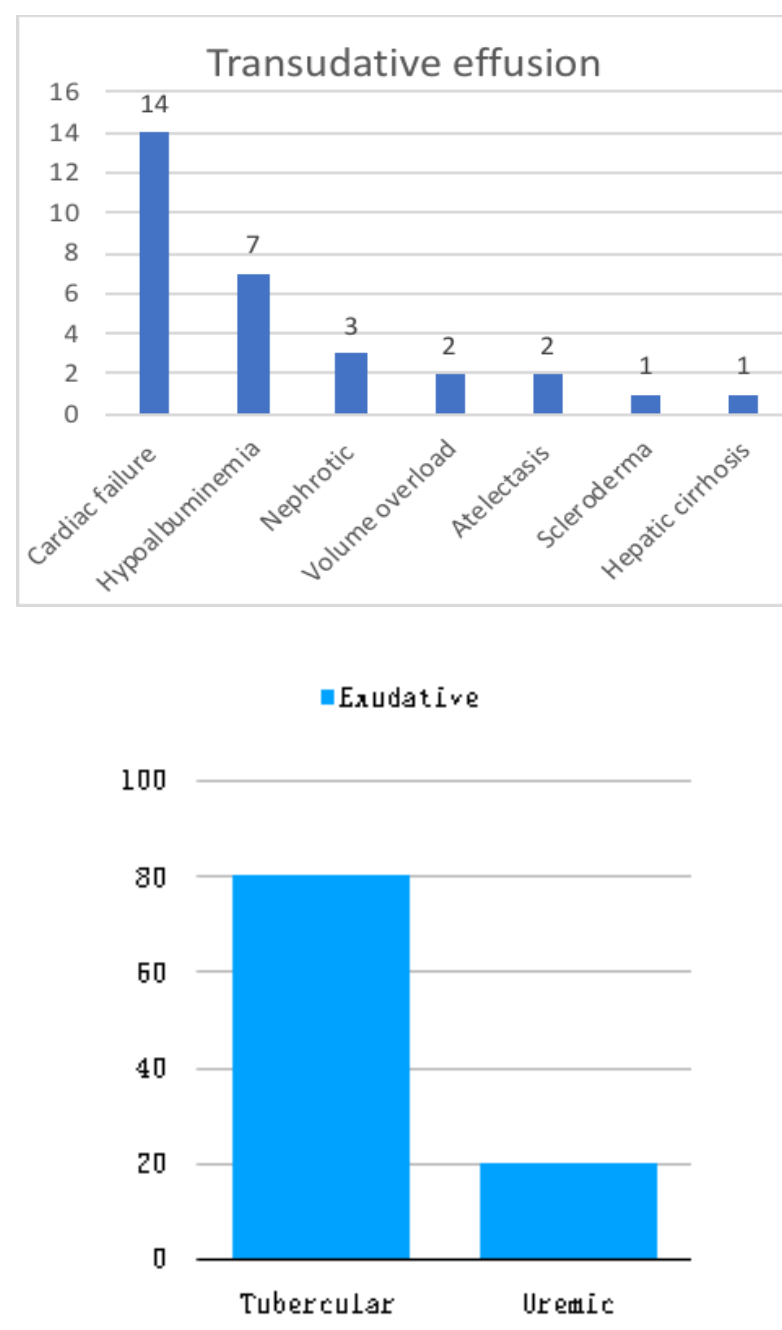

Figure 1. (b) Break up of cases with Type of Pleural effusion

Out of total 10 cases, causes for exudative effusion were tuberculous-8 (80\%), Uremic -2 (20\%). No parapneumonic or empyema cases were noted. 
Most common associated clinical feature was cough (75\%) followed by pleuritic chest pain (55\%); breathlessness (50\%); fever (15\%); night sweats (10\%), weight loss (5\%). Overall prevalence of tuberculous effusion was $20 \%$ among CKD cases. Most common associated comorbidity was hypertension $(67.5 \%)$ followed by diabetes $(52.5 \%)$. However No association was seen between Diabetes and Hypertension with type of pleural effusion ( $\mathrm{p}-0.148$ )

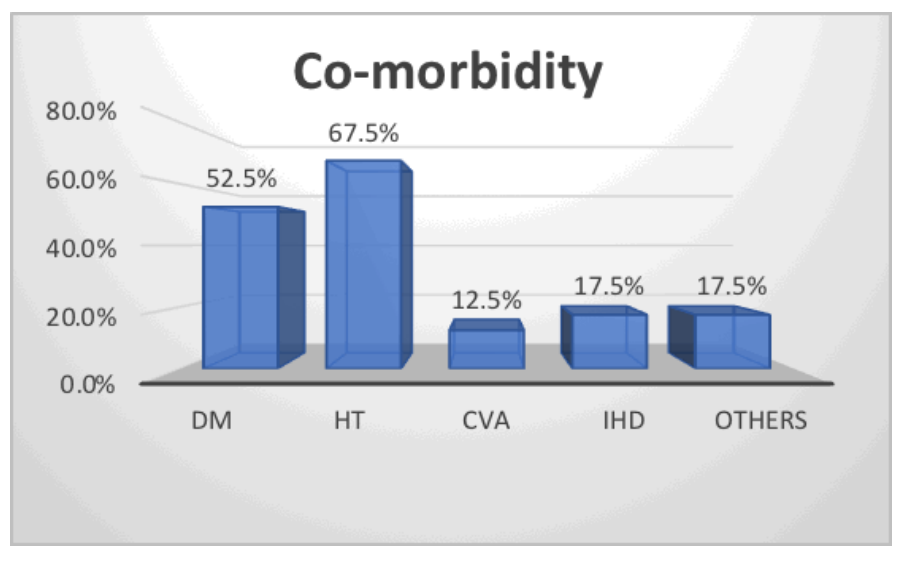

Figure 2

Bilateral pleural effusion was seen in $60 \%$ cases while right and left effusion was seen in $27.5 \%$ and $12.5 \%$ cases respectively. No association was seen between gender and type of pleural effusion (p-0.471).

Transudative effusion was observed to be associated with increasing age $(\mathrm{p}<0.05)$.

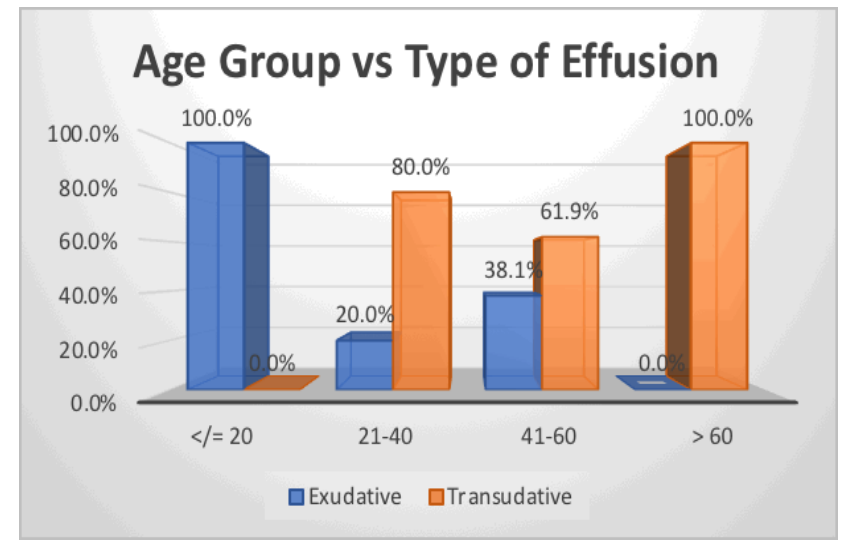

Figure 3

\section{Discussion}

Pleural effusion is the most common pleural disease affecting a significant bulk of population in India. It can be a result of pleural, lung parenchymal, and systemic disease. The pleural effusion may be benign or malignant. ${ }^{(5)}$ The pleural fluid is characterized into transudate and exudate based on the modified Light's criteria. Exudative pleural fluid has at least one of the following characteristics as per the Light's criteria: 1 . Pleural fluid protein/serum protein ratio more than 0.5 ; (2) Pleural fluid lactate dehydrogenase $(\mathrm{LDH}) /$ serum $\mathrm{LDH}$ ratio more than 0.6 and; (3) Pleural LDH is more than twothirds of the upper limits of normal laboratory value for serum $\mathrm{LDH}$. If these criteria are not met, then the fluid is considered as transudate.

The present study aimed to evaluate the spectrum of pleural effusion in patients with chronic kidney disease with special reference to find out the prevalence of tuberculous effusions in patients with CKD.

There are many causes of pleural effusion in uremic patients. In uremic patients, pulmonary edema can occur secondary to a variety of interacting factors such as pulmonary over hydration, left ventricular failure, uremic cardiomyopathy, coronary disease, anaemia or polycythemia, hypoproteinemia, high-output arteriovenous fistula, injury of the alveolocapillary membrane secondary to toxic lesional factors, uremic pleurisy. ${ }^{(7)}$ Uraemic pleurisy is a diagnosis of exclusion that persists or recurs despite aggressive haemodialysis ${ }^{(8)}$

The frequency of bronchopulmonary infections in chronic kidney disease patients is greater than that in the rest of the population due to depression of humoral and cellular immunity and to reduced macrophage activity. The body fluid balance and homeostasis is the most important deciding factor when it comes to chronic kidney disease patients. The thoracic complications of hemodialysis are mainly related to loss in the equilibrium of body 
fluid balance in the extravascular and intravascular compartments. ${ }^{(9)}$

It was observed that mean age of the study subjects was 53.67 years with over half of the cases in the age group of 41-60 years. A male predominance was seen in the study group with $57.5 \%$ males to $42.5 \%$ females which was similar to the study done by Ray $\mathrm{S}$ et al. ${ }^{(8)}$ The age distribution of the patients in the study by Virupakshappa et al. ${ }^{(10)}$ ranged from 15 years to 70 years with maximum number of patients in the age group 41 to 50 years. Average age of study cases was $46 \pm 11.6$ years with 35 males $(70 \%)$ and 15 females $(30 \%)$.

Diabetes and hypertension as co-morbidities was present in $52.5 \%$ and $67.5 \%$ cases of CKD respectively. Other major co-morbidities seen in CKD cases was IHD (30\%) and CVA (12.5\%). This trend is similar to that reported by Dash and Agarwal in the study conducted at the All India Institute of Medical Sciences ${ }^{(11)}$. Lysaght et al have also demonstrated similar trends in American populations $^{(12)}$. In the study conducted by Xue et al the number of patients with diabetic nephropathy was almost $50 \%$ of the study group (13)

Pleural effusion is common in CKD and was observed to be predominantly transudative in most of the studies ${ }^{(14)}$ In present study too, Out of total 40 cases, $30(75 \%)$ had transudative effusion while remaining $10(25 \%)$ had exudative effusion. The aetiological diagnosis in patients with exudative pleural effusion has always been a diagnostic dilemma. This predicament becomes all the more obvious in patients having exudative effusion with co-morbid illnesses like chronic kidney disease (CKD). Patients on dialysis are at a growing risk for tuberculosis (TB) worldwide with a recent summary article documenting a 6.9 to 52.5-fold increased risk. Besides defective-cellmediated immune response and uraemia, comorbid conditions such as diabetes and prolonged corticosteroid and immunosuppressive therapy also predispose patients to active $\mathrm{TB}{ }^{(15)}$. A higher incidence of pulmonary tuberculosis had been reported in patients with CKD on hemodialysis in India, $8.7 \%$ in patients on maintenance hemodialysis and $12.3 \%$ in renal transplant patients ${ }^{(16)}$. In present study, out of the 10 cases of exudative effusion, $8(80 \%)$ were of tuberculous etiology. Overall prevalence of tuberculous effusion was $20 \%$ among CKD cases. In the study by Virupakshappa et al. ,the tuberculous etiology was found in $16 \%$ of patients. Ray et al. in their study observed tuberculous etiology in 26\% CKD cases with pleural effusion. Qureshi SQ et al. ${ }^{(17)}$ in a similar study observed prevalence of tuberculous effusion in CKD cases as 30\%. Management of TB in CKD patients is more complicated than usual TB patient management since most of the drugs except rifampicin requires dose modification and RNTCP regimen is very difficult to administer. Higher incidence of vomiting and gastritis further complicates the compliance for the drugs. Adverse drug effects are more frequent and require a very close follow-up and care.

Jarratt et al found a $21 \%$ incidence of pleural effusion in patients receiving long-term hemodialysis.In our study the most common cause of pleural effusion was heart failure as was observed in a study by Jarratt et al. ${ }^{(18)}$ Infection is the second most common cause of death in patients undergoing long-term hemodialysis, and at autopsy $45 \%$ have pathological evidence of significant acute pulmonary infection. ${ }^{(19)}$

Jarratt et al reported that $15 \%$ of the patients were directly related to pneumonia, and they did not identify any cases of tuberculous pleurisy, on the other hand in our study every 5th patient of chronic kidney disease had a tubercular origin to pleural effusion. The difference may be attributed to this study taken place in India which is a TB endemic nation. However tuberculous pleural effusion is being increasingly recognized, even in developed nations, as the incidence of EPTB has more than doubled following the HIV pandemic. 
Most tuberculous pleural effusion manifest as an acute illness, with approximately one third of patients being symptomatic for $<1$ week and two thirds for $<1$ month. Pleural fluid analysis by a diagnostic pleurocentesis is very important in chronic kidney disease patients.

The definitive diagnosis of TPE depends on the demonstration of Mycobacterium tuberculosis in the sputum, pleural fluid, or pleural biopsy specimens. Other diagnostic methods are by ADA, mycobacterium stain and liquid culture, interferon gamma, interleukin 27 level nucleic acid amplification test and pleural biopsy. ${ }^{(20)}$

To summarize, present study observed that pleural effusion is a common occurrence among patients with CKD. Etiologies of these are multifactorial. Transudative effusions are found to be more common than exudative effusions. In exudative effusions tuberculous pleural effusions were seen predominantly. Tuberculous effusion was observed in every one out of five patients with CKD. We thus recommend that, while evaluating a case of pleural effusion, a combined approach, involving clinical evaluation, radiographic and sonographic evaluation, pleural fluid analysis, pleural fluid cytology, must be utilized for accurate diagnosis, in view of the different management strategies for varied etiologies.

\section{Conclusion}

This study concludes that there are multiple aetiologies for pleural effusion in chronic kidney disease patients and among them, congestive cardiac failure is the most common cause.Invective aetiology for pleural effusion in chronic kidney disease patients undergoing hemodialysis was second most common. Pneumonia and pleural effusion that of tubercular origin should be considered in cases of exudative unilateral and bilateral pleural effusion.

Chronic kidney disease patients should be offered a good cardiac therapy and control of blood pressure. The immune status of these patients should be improved keeping a high index of suspicion for tubercular pleural effusion. If the effusion is found to be exudative, prompt treatment of the condition with the appropriately modified dosages of the drugs should be done.

\section{References}

1. Levey A. S, Eckhardt K U, Tsukamoto Y, et al. Definition and classification of chronic kidney disease: a position statement from kidney disease: Improving Global Outcomes (KIDGO). Kidney international. 2005; 67:2089- 2100

2. Mark Cohen M' Sahn SA. Resolution of pleural effusions. Chest 2001;119:1547-62.

3. Gupta R, Gupta A, Illyas M. Spectrum of pleural effusion etiology revised in $18-70$ years of age group: A tertiary care centre based study of 1000 patients. CHRISMED J Health Res 2018;5: 110-3

4. Ren W, Pan H, Wang P, Lan L, Chen W, Wang Y, Ni L, Peng L. Clinical analysis of pulmonary infection in hemodialysis patients. Experimental and therapeutic medicine. 2014 Jun 1;7(6):1713-7.

5. Charalampidis C, Youroukou A, Lazaridis G, Baka S, Mpoukovinas I, Karavasilis V, Kioumis I, Pitsiou G, Papaiwannou A, Karavergou A, Tsakiridis K. Physiology of the pleural space. Journal of thoracic disease. 2015 Feb;7(Suppl 1):S33.

6. Light RW. The Light criteria: the beginning and why they are useful 40 years later. Clinics in chest medicine. 2013 Mar 1;34(1):21-6.

7. Hooper C, Lee YG, Maskell N. Investigation of a unilateral pleural effusion in adults: British Thoracic Society Pleural Disease Guideline 2010. Thorax. 2010 Aug 1;65(Suppl 2):ii4-17.

8. Ray S, Mukherjee S, Ganguly J, Abhishek K, Mitras S, Kundu S. A cross-sectional prospective study of pleural effusion among cases of chronic kidney disease. Indian $\mathbf{J}$ Chest Dis Allied Sci. 2013;55(4):209-13.

9. Morfin JA, Fluck RJ, Weinhandl ED, Kansal S, McCullough PA, Komenda P. Intensive 
hemodialysis and treatment complications and tolerability. American journal of kidney diseases. 2016 Nov 1;68(5):S43-50.

10. Virupakshappa $\mathrm{V}$ et a. Profile of pleural effusion in chronic kidney disease patients undergoing hemodialysis. Indian Journal of Immunology and Respiratory Medicine, October-December 2017;2(4): 103-107.

11. Dash SC, Agarwal SK. Incidence of chronic kidney disease in India. Nephrology Dialysis Transplantation. 2005 Oct 11;21(1):232-3.

12. Lysaght MJ. Maintenance dialysis population dynamics: current trends and long-term implications. Journal of the American Society of Nephrology. 2002 Jan 1;13(suppl 1):S3740.

13. Xue JL, Ma JZ, Louis TA, Collins AJ. Forecast of the number of patients with endstage renal disease in the United States to the year 2010. Journal of the American Society of Nephrology. 2001 Dec 1;12(12):2753-8.

14. Mogar V, Arun BS, Suresh H, Sagar Reddy SL. A study of respiratory manifestations in chronic kidney disease. International Journal of Biomedical Research. 2017;8(2):70-4.

15. Hussein MM, Mooji JM, Roujouleh $\mathrm{H}$. Tuberculosis and chronic renal disease. Semin Dial 2003;16:38-44.

16. John GT. Infections after renal transplantation in India. Indian J Nephrol. 2003 Jan 1;13:149.

17. Qureshi SQ, Idrees MK, Ahmad S, Ahmed E. Pleural effusion among patients on maintenance hemodialysis at SIUT Karachi, Pakistan. Rawal Medical Journal. 2016 Jan 1;41(1):11-4.

18. Jarratt MJ, Sahn SA: Pleural effusions in hospitalized patients receiving long term hemodialysis. Chest 108:470, 1995

19. 20. Thompson S, James M, Wiebe N, Hemmelgarn B, Manns B, Klarenbach S, Tonelli M. Cause of death in patients with reduced kidney function. Journal of the American Society of Nephrology. 2015 Oct 1;26(10):2504-11.
20. Ocaña I, Martinez-Vazquez JM, Segura RM, Fernandez-De-Sevilla T, Capdevila JA. Adenosine deaminase in pleural fluids: test for diagnosis of tuberculous pleural effusion. Chest. 1983 Jul 1;84(1):51-3. 